\title{
Analysis of Mutation Rate of 17 Y-Chromosome Short Tandem Repeats Loci Using Tanzanian Father-Son Paired Samples
}

\author{
Fidelis Charles Bugoye $\mathbb{D}^{1}{ }^{1}$ Elias Mulima, ${ }^{1}$ and Gerald Misinzo ${ }^{2}$ \\ ${ }^{1}$ Department of Forensic Science and DNA Services, Government Chemist Laboratory Authority, Dar es Salaam, Tanzania \\ ${ }^{2}$ Department of Veterinary Microbiology, Parasitology and Biotechnology, Sokoine University of Agriculture, Morogoro, Tanzania \\ Correspondence should be addressed to Fidelis Charles Bugoye; bugoye81@yahoo.co.uk
}

Received 12 April 2018; Accepted 24 July 2018; Published 6 August 2018

Academic Editor: Giuseppe Novelli

Copyright (c) 2018 Fidelis Charles Bugoye et al. This is an open access article distributed under the Creative Commons Attribution License, which permits unrestricted use, distribution, and reproduction in any medium, provided the original work is properly cited.

\begin{abstract}
Hundred unrelated father-son buccal swab sample pairs collected from consented Tanzanian population were examined to establish mutation rates using 17 Y-STRs loci DYS19, DYS389I, DYS389II, DYS390, DYS391, DYS392, DYS393, DYS385a, DYS385b, DYS437, DYS438, DYS439, DYS448, DYS456, DYS458, DYS635, and Y-GATA-H4 of the AmpFISTRYfiler kit used in forensics and paternity testing. Prior to 17 Y-STRs analysis, father-son pair biological relationships were confirmed using 15 autosomal STRs markers and found to be paternally related. A total of four single repeat mutational events were observed between father and sons. Two mutations resulted in the gain of a repeat and the other two resulted in a loss of a repeat in the son. All observed mutations occurred at tetranucleotide loci DYS389II, DYS385a, and DYS385b. The locus specific mutation rate varied between 0 and $1.176 \times 10^{-3}$ and the average mutation rate of $17 \mathrm{Y}$-STRs loci in the present study was $2.353 \times 10^{-3}\left(6.41 \times 10^{-4}-6.013 \times 10^{-3}\right)$ at $95 \%$ CI. Furthermore the mean fathers' age with at least one mutation at son's birth was 32 years with standard error of 2.387 while the average age of all fathers without mutation in a sampled population at son's birth was 26.781 years with standard error of 0.609 . The results shows that fathers' age at son's birth may have an effect on Y-STRs mutation rate analysis, though this age difference was statistically not significant using unpaired samples t-test $(\mathrm{p}=0.05)$. As a consequence of observed mutation rates in this study, the precise and reliable understanding of mutation rate at Y-chromosome STR loci is necessary for a correct evaluation and interpretation of DNA typing results in forensics and paternity testing involving males. The criterion for exclusion in paternity testing should be defined, so that an exclusion from paternity has to be based on exclusion constellations at a minimum of two 17 Y-STRs loci.
\end{abstract}

\section{Introduction}

Research and application of Y-chromosome short tandem repeats (Y-STRs) have proven beneficial in a number of fields including paternity, anthropology, and genealogical studies [1]. The very useful application of Y-STR systems is due to their potential in detecting and discriminating male DNA. Human Y-STR polymorphisms or microsatellites are useful in resolving and relating male lineages in forensics especially in sexual assault cases where there is a large proportion of mixed male/female stains [2], genealogical [3], evolutionary studies [4], and anthropological applications [5].

The interpretation of DNA evidence in forensic analysis and paternity testing is based on the similarities or differences at a genetic loci used. In parenthood testing, the difference at inheritable genetic marker loci between the putative father and the offspring is attributed to nonbiological paternity and therefore leads to exclusion of biological paternity. On the other hand, the spontaneous mutations in the germline of the putative father at any genetic marker locus used in the analysis can lead to an erroneous exclusion because such mutation results in differences between the parent and offspring. Since new alleles occur due to the mutation events, there is natural correlation between the degree of polymorphism and the underlined mutations rate of any given locus; i.e., the higher the mutation rate is, the more variable the locus is [6].

In forensic DNA typing applications, highly polymorphic loci are usually preferred due to their high power of discrimination. Therefore, short tandem repeat (STR) loci or microsatellites are considered to be the markers of choice in forensics because of their high power of discrimination 
TABLE 1: Mutation count and Y-STRs loci mutation characteristics events as revealed by direct observation on father-son paired samples of previously confirmed biological relationship.

\begin{tabular}{|c|c|c|c|c|c|c|}
\hline Sample ID's & Loci & Repeat sequence $^{\mathrm{a}}$ & Father's profile & Son's profile & Mutation characteristics & Mutation count \\
\hline $\mathrm{F} / \mathrm{C} 003$ & DYS385b & GAAA & 16 & 17 & Gain & 1 \\
\hline $\mathrm{F} / \mathrm{C} 012$ & DYS385a & AAGG & 15 & 16 & Gain & 1 \\
\hline $\mathrm{F} / \mathrm{C} 074$ & DYS385a & AAGG & 16 & 15 & Loss & 1 \\
\hline $\mathrm{F} / \mathrm{C} 082$ & DYS389II & CTGT/CTAT & 31 & 30 & Loss & 1 \\
\hline
\end{tabular}

${ }^{a}$ Repetitive sequence structure previously reported by Gusmao and Carracedo (2003).

and ease of analysis. For criminal and paternity testing investigations, which involve males with deceased alleged father, Y-STRs are used as the marker of choice [7]. YSTRs are preferred because they are transmitted without recombination from fathers to sons and therefore are able to characterise paternal pedigree. In addition, Y-STRs are suitable for sexual assault investigations as they provide male specific DNA profiles which avoid problems of mixed stain interpretation [1]. However, since highly polymorphic YSTR loci applied in forensic investigations constantly evolve through mutations, the evaluation and interpretation of the genetic profiles requires precise knowledge on mutation rates at each loci used. Reliable estimations of mutation rates for these loci are valuable in assisting the interpretation of Y-STRs test results. Most of investigations have reported mutation rates for the minimal haplotype loci in different populations, but very few articles have reported results with the 17 Y-STRs loci mutation rates using African populations.

\section{Materials and Methods}

During this study, buccal swab samples were collected from consented father-son paired samples whose biological relationship was confirmed by autosomal STRs using AmpFISTR Identifiler kit [8]. A total of 100 father-son pairs from Tanzanian population were collected in Dar es Salaam after obtaining informed consent for participation in the study. DNA extraction was done using Chelex method and the extracted DNA were amplified using 17Y-STRs of AmpFISTRYfiler ${ }^{\text {TM }}$ kit (DYS456, DYS389I, DYS390, DYS389II, DYS458, DYS19, DYS385a, DYS385b, DYS393, DYS391, DYS439, DYS635, DYS392, Y-GATA-H4, DYS437, DYS438, and DYS448) [8] using the following conditions: PCR amplification of the Y filer loci was performed using $0.5-1 \mathrm{ng}$ of DNA template and the total $25 \mu \mathrm{L}$ reaction volumes of PCR amplification were used, as recommended by the manufacturer. The PCR amplicons were analyzed using capillary electrophoresis in an ABI Prism 3130xl genetic analyser [8]. Analysis of DNA fragments was performed using a Gene Mapper IDv.3.2 [8]. Data analysis was carried out using the Excel statistical Software [9], and the confidence interval (CI) was estimated from the binomial standard deviation [10].

\section{Results and Discussion}

3.1. 17 Y-STRs Locus Specific Mutation Characteristics. Analysis of locus specific mutation characteristics using 17 Y-STRs loci in Tanzanian father-son pairs of DNA confirmed biological paternity revealed four mutations events which were identified on DYS385a, DYS385b, and DYS389II among 17Y-STRs loci analyzed [9] (Table 1).

However, no mutation event was observed for DYS19, DYS389I, DYS390, DYS391, DYS392, DYS393, DYS437, DYS348, DYS439, DYS448, DYS456, DYS458, DYS635, and Y-GATA-H4 loci analyzed. The observed locus specific mutation rate ranged between 0 for DYS19, DYS389I, DYS390, DYS391, DYS392, DYS393, DYS437, DYS348, DYS439, DYS448, DYS456, DYS458, DYS635, and Y-GATAH4 loci and $1.765 \times 10^{-3}\left(1.43 \times 10^{-4}-4.243 \times 10^{-3}\right)$ for DYS385a locus at 95\% CI [9]. Among 100 father-son pairs analyzed at the same 17 Y-STRs loci, there was no observation of multiple Y-chromosome microsatellite mutation within the same germline transmission or nonuniform alleles such as microvariants, duplication, and triplication that have been previously reported by Laouina [11] in Moroccan population.

The highly polymorphic Y-STR locus DYS385 was observed to have a higher mutation rate compared to all other Y-STRs loci analyzed (Table 2). In this study, the observed higher specific locus mutation rate for Y-STR locus DYS385a/b (if treated as single locus) was $1.765 \times 10^{-3}$ followed by mutation rate of $5.88 \times 10^{-4}$ for locus 389II [9].

All observed mutation events were characterised by single-step mutations (Table 1), in accordance with the generally accepted mutation model for microsatellites, in which the alleles are known to mutate primarily through the gain and loss of single repeat units $[12,13]$.

In addition, two tetranucleotide microsatellites loci DYS385 and 389II appeared to consist of higher average mutation rates among all 17 Y-STRs analyzed compared to all other trinucleotide and dinucleotide microsatellite loci [9]. Similar locus specific mutation characteristics were found in Moroccan's population in a sample of 252 father-son pairs using 17 Y-STRs loci by Laouina [11] in which average locus specific mutation rate was higher at tetra nucleotide microsatellites loci. This higher mutation rate on tetranucleotide microsatellites was also observed by Kayser et al. [1] using 15 Y-STRs loci for a total of 4999 male germline transmission from father-son pairs of previously confirmed paternity. Furthermore, the single loss mutation characteristics event observed in this study was in agreement with research results found by Farfán and Prieto [14] where three single-step loss mutations were observed at DYS389II loci, during mutations analysis at 17 Y-STR loci in father-son pairs from southern Spain. 
TABLE 2: Mutation count, mutation rate and 95\% confidence interval (CI) for the 17 Y-STRs loci studied using Tanzanian father-son paired samples.

\begin{tabular}{|c|c|c|c|c|c|}
\hline Loci & Repetitive DNA sequence $^{a}$ & Mutation count & Allele transmission & Mutation rate & $95 \% \mathrm{CI}$ \\
\hline DYS19 & CTAT/CTAC & 0 & 1700 & 0.000 & $0.000-2.168 \times 10^{-3}$ \\
\hline DYS389I & AAGG/GAAA & 0 & 1700 & 0.000 & $0.000-2.168 \times 10^{-3}$ \\
\hline DYS389II & CTGT/CTAT & 1 & 1700 & $5.88 \times 10^{-4}$ & $1.5 \times 10^{-5}-3.273 \times 10^{-3}$ \\
\hline DYS390 & CTGT/CTAT & 0 & 1700 & 0.000 & $0.000-2.168 \times 10^{-3}$ \\
\hline DYS391 & CTGT/CTAT & 0 & 1700 & 0.000 & $0.000-2.168 \times 10^{-3}$ \\
\hline DYS392 & ATT & 0 & 1700 & 0.000 & $0.000-2.168 \times 10^{-3}$ \\
\hline DYS393 & GATA & 0 & 1700 & 0.000 & $0.000-2.168 \times 10^{-3}$ \\
\hline DYS385a & AAGG & 2 & 1700 & $1.176 \times 10^{-3}$ & $1.43 \times 10^{-4}-4.243 \times 10^{-3}$ \\
\hline DYS385b & GAAA & 1 & 1700 & $5.88 \times 10^{-4}$ & $1.5 \times 10^{-5}-3.273 \times 10^{-3}$ \\
\hline DYS438 & TTTTC/TTTTA & 0 & 1700 & 0.000 & $0.000-2.168 \times 10^{-3}$ \\
\hline DYS439 & GATA & 0 & 1700 & 0.000 & $0.000-2.168 \times 10^{-3}$ \\
\hline DYS437 & TCTA/TCTG & 0 & 1700 & 0.000 & $0.000-2.168 \times 10^{-3}$ \\
\hline DYS448 & AGAGAT & 0 & 1700 & 0.000 & $0.000-2.168 \times 10^{-3}$ \\
\hline DYS458 & GAAA & 0 & 1700 & 0.000 & $0.000-2.168 \times 10^{-3}$ \\
\hline DYS456 & AGAT & 0 & 1700 & 0.000 & $0.000-2.168 \times 10^{-3}$ \\
\hline DYS635 & TCTA/TGTA & 0 & 1700 & 0.000 & $0.000-2.168 \times 10^{-3}$ \\
\hline Y GATA H4 & TAGA & 0 & 1700 & 0.000 & $0.000-2.168 \times 10^{-3}$ \\
\hline Average & & 4 & 1700 & $2.353 \times 10^{-3}$ & $6.41 \times 10^{-4}-6.013 \times 10^{-3}$ \\
\hline
\end{tabular}

3.2. Analysis of 17 Y-STRs Locus Specific Mutation Rate. Using 100 Tanzanian father-son paired samples with confirmed paternity covering 1700 meioses were used to estimate $17 \mathrm{Y}$ STRs locus specific mutation rate. The observed average estimates of 17 Y-STRs locus specific mutation rate ranged from 0 to $1.765 \times 10^{-3}\left(1.43 \times 10^{-4}-4.243 \times 10^{-3}\right)$ at $95 \% \mathrm{CI}$. The higher average locus specific mutation rate was found at DYS385a locus while mutation rates of $5.88 \times 10^{-4}\left(1.5 \times 10^{-5}\right.$ $3.273 \times 10^{-3}$ ) at $95 \%$ CI were observed for both DYS385b and DYS389II loci. There was no mutation observed for DYS19, DYS389I, DYS390, DYS391, DYS392, DYS393, DYS437, DYS348, DYS439, DYS448, DYS456, DYS458, DYS635, and Y-GATA-H4 loci analyzed [9].

The average mutation rate across all markers in this study was $2.353 \times 10^{-3}\left(6.41 \times 10^{-4}-6.013 \times 10^{-3}\right)$ at $95 \%$ CI [9] (Table 2). This overall average mutation rate is nearly similar to those reported by Sanchez-Diz [15] in which a mutation rate of $2.2 \times 10^{-3}$ in 701 father-son pairs in Iberian and Latin America groups was found. Dupuy et al. [16] used 1766 fatherson pairs to analyze 9Y-STRs alone in Norway's population and found the average mutation rate of $2.3 \times 10^{-3}$. A similar mutation rate of $2.3 \times 10^{-3}$ was found by Lee et al. [17] in collection of Y-STRs mutation events for Korean population using a high number of loci, 22 Y-STRs in a sample size equal to 369 father-son pairs. Furthermore, the average mutation rate estimated in this study is not significantly different from the average mutation rate of autosomal STR loci commonly used in forensics as previously reported from family analysis. In their findings, the autosomal STRs mutation rate of 2.1 $\times 10^{-3}$ was reported by Brinkman et al., 1998; mutation rate of $2.7 \times 10^{-3}$ was reported by Henke and Henke [18] whereas mutation rate of $0.6 \times 10^{-3}$ was reported by Sajantila [19].
The average mutation rate for 17Y-STRs loci found in this research study is greater than those calculated by Viera-Silva [20] in a sample of 95 father-son pairs from Portugal $(1.85 \times$ $10^{-3}$ ) and by Farfán and Prieto [14] using 17 Y-STRs loci from southern Spain population was $1.563 \times 10^{-3}\left(0.322 \times 10^{-3}\right.$ $\left.4.559 \times 10^{-3}\right)$ at $95 \% \mathrm{CI}$, but the average mutation rate found in this study is less than those calculated by Decker [21] for Caucasian and Asians populations cited up; in US admixed sample of 399 father-son pairs using 17 Y-STRs, the mutation rate found was $3.13 \times 10^{-3}$.

The results of present study are in general agreement with the fore mentioned research findings in which all the same 17 Y-STRs set or other number Y-STRs loci used the average mutation rate observed were in the order of $10^{-3}$ though number of father-son pairs varied between the mentioned studies above. Since there were no significant differences in mutation rate observed, therefore the mutation rates analysis does not depend on the sample size or number of Y-STRs loci used but population diversity [9].

3.3. Effects of Father's Age on 17 Y-STR Mutation Rate Analysis. The present study shows that the average fathers' age with at least one mutation at son's birth was 32 years with standard error of 2.387 while the average age of all fathers without mutation in a sampled population at son's birth was 26.781 years with standard error of 0.609 (Table 3). Results shows undoubtedly the age of the mutated father from our study which is marginally older than that without mutations. The results clearly shows that fathers' age at son's birth may have an effect on Y-STRs mutation rate analysis, though this age difference is statistically not significant using unpaired samples t-test $(p=0.05)$ [9]. The results of present study are in agreement with results of research findings by Sanchez-Diz 
TABLE 3: Fathers's age at the time of Sons' birth with at least one mutation and without any mutation on 17 Y-STRs loci.

\begin{tabular}{ll}
\hline Fathers' age (Years) with at least one mutation & Fathers' age (Years) without any mutation \\
\hline $25,40,32,31$ & $19,20,26,25,30,21,25,26,24,30,32,30,21,28,30,30,24,25,34,20$ \\
& $35,18,19,18,19,20,32,36,32,33,34,32,21,20,25,34,29,28,35,25$ \\
& $24,26,25,24,26,25,34,23,25,24,28,29,25,26,23,24,27,28,36,26$ \\
& $37,25,34,20,34,18,19,18,19,39,32,33,32,33,36,32,42,34,20,18$ \\
\hline Average $=\mathbf{3 2 . 0 0 0}$ & $18,19,26,19,20,32,33,32,33,36,25,20,24,18,29,19$ \\
Standard error $=\mathbf{2 . 3 8 7}$ & Average $=\mathbf{2 6 . 7 8 1}$ \\
$\mathbf{P}$ Value $=\mathbf{0 . 0 6 0}$ & Standard error $=\mathbf{0 . 6 0 9}$ \\
\hline
\end{tabular}

[15], Lee [17], and Goebloed [22] who also reported relatively older average age of mutated fathers in their studies but the age difference between fathers' age with at least one mutation and fathers' age without mutation was found statistically not significant.

\section{Conclusion}

The results of 17-Y-STRs mutation observed from this study revealed that the precise and reliable understanding of mutation rate at Y-chromosome short tandem repeats loci is necessary for a correct evaluation and interpretation of DNA typing results in forensics and paternity testing involving males. Based on the findings, the criterion for exclusion in paternity testing should be defined in any DNA testing laboratory using 17-Yfiler Amplification kits, so that an exclusion from paternity has to be based on exclusion constellations at the minimum of two 17 Y-STRs loci.

\section{Data Availability}

The mutation data used to support the findings of this study are included within the articles. The generated Sons' Y-STRs haplotype data were only submitted to YHRD (http://www.yhrd.org/) and received the Accession no. YC000312.

\section{Ethical Approval}

The study and permission to publish have been approved by Medical Research Coordinating Committee (MRCC) of National Institute for Medical Research (NIMR), Tanzania. Permit nos. NIMR/HQ/R.8a/VOL.IX/1826 and NIMR/HQ/P.12 VOLXIX/34 were obtained.

\section{Consent}

Informed consent was obtained from all individual participants included in the study.

\section{Disclosure}

The analyses were carried out at forensic biology and DNA laboratory, Government Chemist Laboratory Authority.

\section{Conflicts of Interest}

The authors declare that they have no conflicts of interest.

\section{Acknowledgments}

This study was funded by the Government Chemist Laboratory Authority (GCLA). Fidelis Charles Bugoye is a recipient of a scholarship from GCLA through technical capacity training program.

\section{References}

[1] M. Kayser, P. De Knijff, P. Dieltjes et al., "Application of microsatellite-based Y chromosome haplotyping," Electrophoresis, vol. 18, no. 9, pp. 1602-1607, 1997.

[2] A. J. Redd, A. B. Agellon, V. A. Kearney et al., "Forensic value of 14 novel STRs on the human Y chromosome," Forensic Science International, vol. 130, no. 2-3, pp. 97-111, 2002.

[3] M. Kayser, M. Vermeulen, H. Knoblauch, H. Schuster, M. Krawczak, and L. Roewer, "Relating two deep-rooted pedigrees from Central Germany by high-resolution Y-STR haplotyping," Forensic Science International: Genetics, vol. 1, no. 2, pp. 125-128, 2007.

[4] M. A. Jobling and C. Tyler-Smith, "The human Y chromosome: An evolutionary marker comes of age," Nature Reviews Genetics, vol. 4, no. 8, pp. 598-612, 2003.

[5] P. De Knijff, "Messages through bottlenecks: On the combined use of slow and fast evolving polymorphic markers on the human Y chromosome," American Journal of Human Genetics, vol. 67, no. 5, pp. 1055-1061, 2000.

[6] M. Kayser and A. Sajantila, "Mutations at Y-STR loci: Implications for paternity testing and forensic analysis," Forensic Science International, vol. 118, no. 2-3, pp. 116-121, 2001.

[7] L. Roewer, M. Kayser, P. De Knijff et al., "A new method for the evaluation of matches in non-recombining genomes: Application to Y-chromosomal short tandem repeat (STR) haplotypes in European males," Forensic Science International, vol. 114, no. 1, pp. 31-43, 2000.

[8] AmpFlSTR_Yfiler_PCR Amplification Kit User Guide, Applied Biosystems, Foster City, CA, USA, 2006.

[9] F. Charles, Analysis of Mutation Rate of 17 Y-Chromosome Short Tandem Repeats Loci Using Tanzanian Father-Son Paired Samples, 2015, suanet.ac.tz: 8080/xmlui/handle/123456789/1290., http://www.suaire.

[10] http://statpages.org/confint.html. 
[11] A. Laouina, S. Nadifi, R. Boulouiz et al., "Mutation rate at 17 Y-STR loci in "Father/Son" pairs from moroccan population," Legal Medicine, vol. 15, no. 5, pp. 269-271, 2013.

[12] J. L. Weber and C. Wong, "Mutation of human short tandem repeats," Human Molecular Genetics, vol. 2, no. 8, pp. 1123-1128, 1993.

[13] L. A. Zhivotovsky and M. W. Feldman, "Microsatellite variability and genetic distances," Proceedings of the National Acadamy of Sciences of the United States of America, vol. 92, no. 25, pp. 11549-11552, 1995.

[14] M. J. Farfán and V. Prieto, "Mutations at 17 Y-STR loci in fatherson pairs from Southern Spain," Forensic Science International: Genetics Supplement Series, vol. 2, no. 1, pp. 425-426, 2009.

[15] P. Sánchez-Diz, C. Alves, E. Carvalho et al., "Population and segregation data on 17 Y-STRs: Results of a GEP-ISFG collaborative study," International Journal of Legal Medicine, vol. 122, no. 6, pp. 529-533, 2008.

[16] B. M. Dupuy, M. Stenersen, T. Egeland, and B. Olaisen, "YChromosomal Microsatellite Mutation Rates: Differences in Mutation Rate between and Within Loci," Human Mutation, vol. 23, no. 2, pp. 117-124, 2004.

[17] H. Y. Lee, M. J. Park, U. Chung et al., "Haplotypes and mutation analysis of 22 Y-chromosomal STRs in Korean father-son pairs," International Journal of Legal Medicine, vol. 121, no. 2, pp. 128135, 2007.

[18] J. Henke and L. Henke, "Mutation rate in human microsatellites," American Journal of Human Genetics, vol. 64, no. 5, pp. 1473-1474, 1999.

[19] A. Sajantila, M. Lukka, and A.-C. Syvänen, "Experimentally observed germline mutations at human micro- and minisatellite loci," European Journal of Human Genetics, vol. 7, no. 2, pp. 263266, 1999.

[20] C. Vieira-Silva, P. Dario, T. Ribeiro, I. Lucas, H. Geada, and R. Espinheira, "Y-STR mutational rates determination in South Portugal Caucasian population," Forensic Science International: Genetics Supplement Series, vol. 2, no. 1, pp. 60-61, 2009.

[21] A. E. Decker, M. C. Kline, J. W. Redman, T. M. Reid, and J. M. Butler, "Analysis of mutations in father-son pairs with 17 Y-STR loci," Forensic Science International: Genetics, vol. 2, no. 3, pp. e31-e35, 2008.

[22] M. Goedbloed, M. Vermeulen, R. N. Fang et al., "Comprehensive mutation analysis of $17 \mathrm{Y}$-chromosomal short tandem repeat polymorphisms included in the AmpFSTR ${ }^{\circledR}$ Yfiler $^{\circledR}$ PCR amplification kit," International Journal of Legal Medicine, vol. 123, no. 6, pp. 471-482, 2009. 


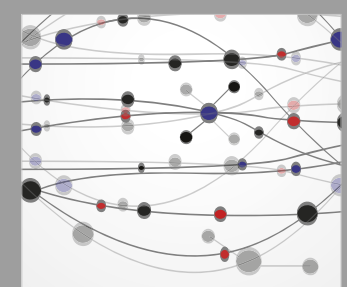

The Scientific World Journal
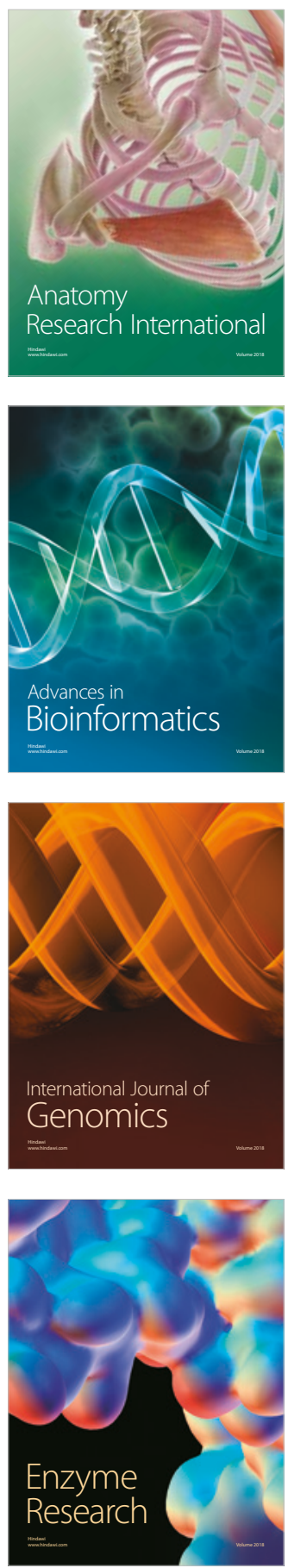
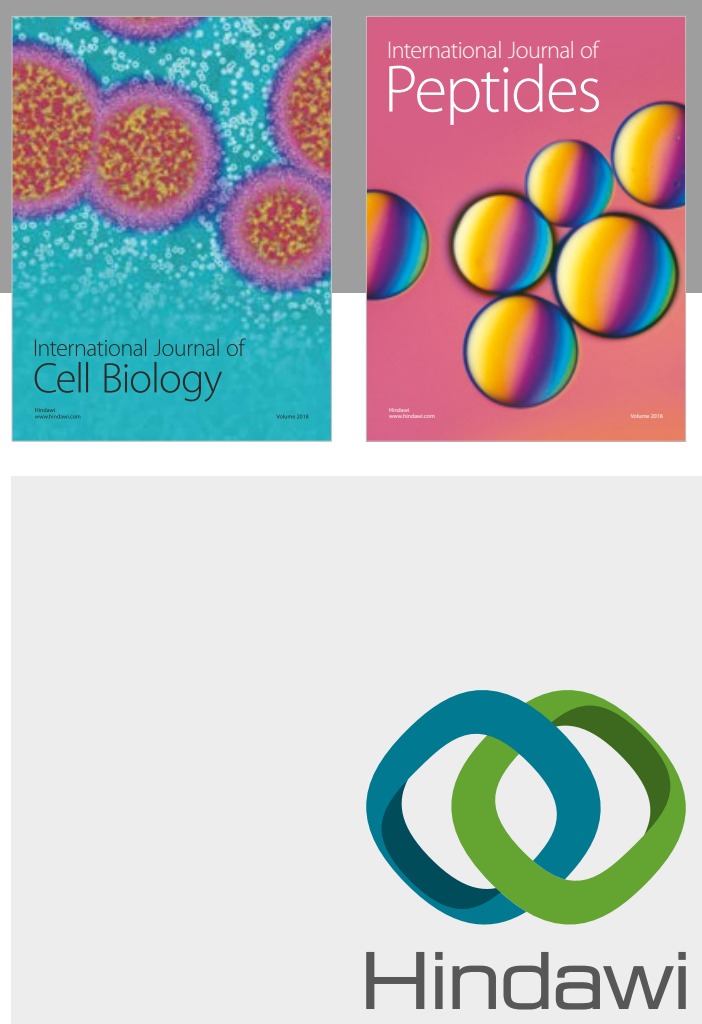

Submit your manuscripts at

www.hindawi.com
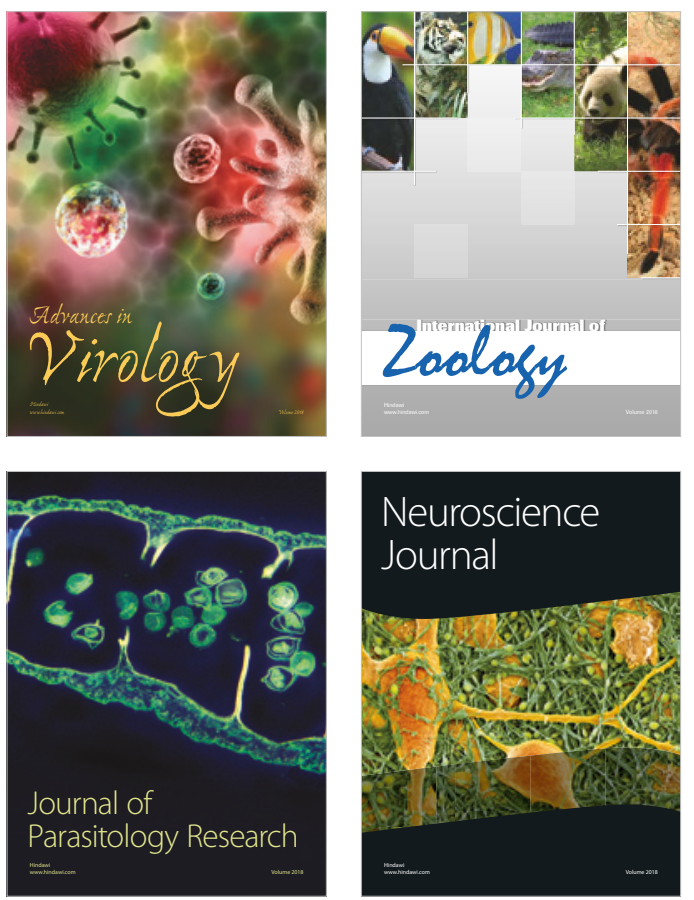
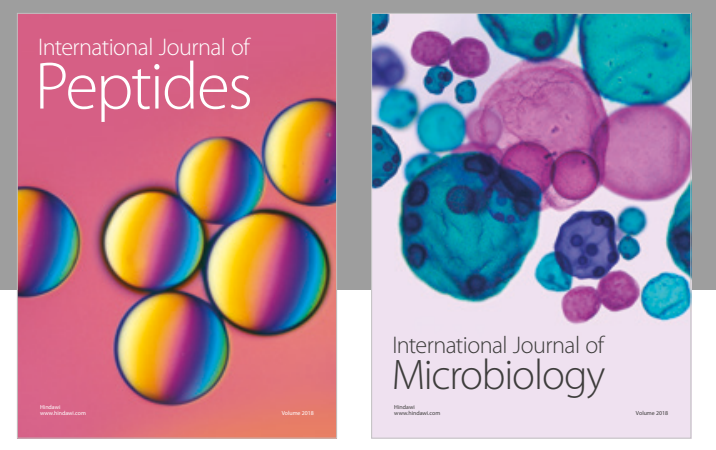

nternational Journal of Microbiology
Journal of
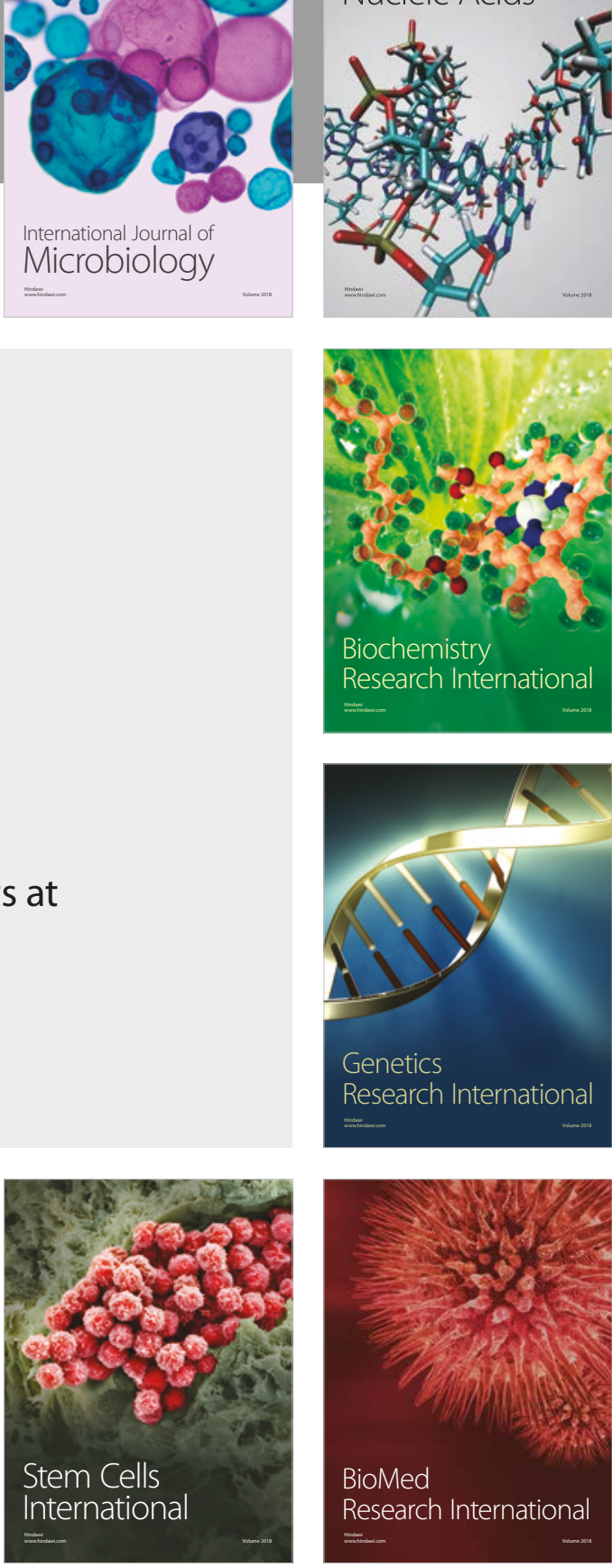
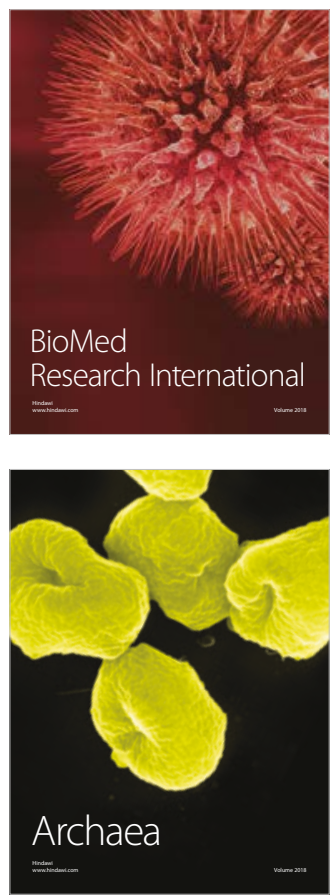